\title{
Index des noms de personne
}

\begin{tabular}{rlcl}
\multicolumn{3}{c}{ Abréviations } \\
anc. $=$ ancien & helv. $=$ helvétique \\
cap. $=$ capitaine & prés. $=$ président \\
chât. $=$ châtelain & prof. $=$ professeur \\
dép. $=$ député & rég. $=$ régiment \\
diz. $=$ dizain & trib. $=$ tribunal \\
gén. $=$ général & val. $=$ valaisan
\end{tabular}

A

Abbatucci, v. Huningue

Aberdeen, George-Hamilton-Gordon (17841860), comte $d^{\prime}-$, ambassadeur d'Angleterre à Vienne : 33

Achard-James, Jean-Marie (1780-1848), conseiller auditorial à la cour impériale de Lyon : 23

Addington, Henry-U. (1757-1844), vicomte Sidmouth, premier secrétaire de la légation anglaise en Suisse : 90, 107

Affry, Charles (1772-1818), comte d'-, colonel et inspecteur des troupes suisses $1815: 260$

Alexandre le Grand : 62

Alexandre Ier, empereur de Russie 1801$1825: 28,33,35,55,61,202$

Allet, Eugène (1784-1837), dép. à la Diète val. : 173

Amacker, Christian (Johann-) (1785-1862), anc. chât. de Rarogne, dép. à la Diète val. : 172

Ambuel, Alphonse († 1801), grand chât. de Sion : 144

Amherd, Aloïs (1755-1825), curé de Sion 1811-1816 : 201

Andenmatten, Donat (1786-1841), greffier du diz. de Viège : 172

Anderledy, huissier (sautier) des dép. val. à la Diète fédérale : 235

Artois, comte $\mathrm{d}^{\prime}-$, frère de Louis XVIII, colonel-général des Suisses : $36,60-61$, 87,265
B

Bachmann, Niklaus-Franz (1740-1831), gén. en chef des troupes suisses : 239, 242245, 248-249, 252, 254-255, 257-258, 260

Barberini, Emmanuel (Joseph-) (1733-1807), banneret de Sion : 143

Beauvoir, agent d'affaires à Lyon : 218

Beeger, Maurice, de Sierre : 81

Bellegarde, Henri (1756-1846), comte de -, gén. gouverneur des possessions autrichiennes en Italie : 228, 247

Berclaz, Marie-Josèphe, née Walter, sagefemme, veuve du chirurgien François Berclaz († 1814) : 37

Blücher, Gerhardt Leberecht (1742-1819) von -, gén. prussien : 31, 34, 249

Bonaparte, Joseph-Napoléon, ex-roi d'Espagne : 83

- Napoléon, v. Napoléon Ier

Bonivini, Adrien (-Pierre), de Sierre : 81, 210-211, 213

Bonjean, Emmanuel (1795-1841), de Vouvry : 184

Bons, Charles (-Louis) (1756-1841) de -, colonel : 139

Bontems, Charles-Henri (1786-1842), aide de camp du colonel von Sonnenberg: 245

Borrat, Mathias : 233

Bourbons : $36,60,135$

Bovier, Charles (1787-1863), de Vex, avocat et notaire : 204 
Bruttin : 204

Bubna, Ferdinand (1768-1825), comte de - , feld-maréchal autrichien : 122, 242, 249

Bürcher, Jean-Joseph, grand chât. de Conches : 170

Bürkli, Johann-Heinrich (1760-1821), rédacteur de la Wochenzeitung, à $\mathrm{Zu}-$ rich : 81

Buxtorf, Andreas (1740-1815) commissaire du Directoire helv. en Valais 1799 : 99

C

Canning, Stratford, ministre de GrandeBretagne en Suisse 1814-1820 : 47-52, $55,57,61,65,68-69,76-79,86,90,107$, 130-131, 146, 149-150, 199-200, 204, 235-238, 244, 246

Capo d'Istria, Jean-Antoine, ministre plénipotentiaire d'Alexandre Ier près la Diète helv. 1814-1815: 43, 50-51, 5456, 61-62, 107, 118, 146, 199-200, 202, $204,235,238$

Capol, baron de -, major au rég. de Roll : 67,70

Castella, Nicolas-Antoine-Xavier (17671830), commandant en second de l'armée suisse 1815: 255, 260

Castlereagh, Robert Stewart, lord -, ministre des Affaires étrangères de Grande-Bretagne : 33,90

Catherine, servante chez Eug. de Courten, à Sierre : 50, 63, 72, 74, 79

Chambrier, Jean-Pierre (1753-1822) de -, ministre de Prusse en Suisse 1814: 38, 118

Chappex, Jean-Louis, de Finhaut : 139

Charles, prince (1771-1847), archiduc d'Autriche : 36

Charles-Louis, grand-duc de Bade 18111818: 28-29

Chastonay, François-Nicolas de -, grand chât. de Sierre : 79, 81, 211

Claivaz, Etienne (-Joseph) (1772-1842), grand chât. de Martigny: 142, 145, 147-149, 206, 232

Cocatrix, Xavier (Jacques-François-) (17891862), secrétaire du conseil de diz. de Saint-Maurice : 139

Coquoz, Nicolas, conseiller, de Salvan : 139

Courten, famille de - : 211

- Adolphe (Eugène-Louis-) (1812-1892) de -, fils d'Eugène : 58,63

- Adrien (Joseph - $A^{\prime}$ - Eugène - Antoine) (1771-1835) de -, fils de François-Antoine : 82
- Amand (Jean-Joseph-) (1773-1851) de -, officier au rég. de Watteville : 72

- Anne-Marie (1776-1846) de -, fille de Joseph-Eugène-Adrien, belle-sœur $d^{\prime} E u-$ gène et de Pancrace : 64

- Antoine-Adrien (Jean-) (1725-1803) de -, oncle d'Eugène et de Pancrace, à Sierre : 74

- Augustine-Louise-Angélique (1813-1820) de -, fille d'Amand : 72

- Catherine (Marie-C'-Antoinette) (17681847) de -, sœur d'Amand : 72

- Eugène (1771-1839) de -, comte, fils de Pancrace, dép. à Fribourg-en-Brisgau, dép. à Zurich, colonel fédéral : 24-38, 46-90, 94, 109, 111, 117, 205, $224,228,239,245,262,267$

- Eugénie (Marie-Anne-) (1774-1814) de -, fille de Joseph-Eugène-Adrien et de Marie-Jeanne Duchemin, épouse (1798) $d^{\prime}$ Eugène de Courten : 26, 28-30, 52-53, $56,59-60,63-65,71$

- Fanie (Marie-Elisabeth-Françoise) (1775 -1835) de -, dite -, fille de JosephEugène-Adrien et de Marie-Jeanne Duchemin, épouse (1803) de Pancrace de Courten : 35, 37, 53, 82

- Jeanne (Marie-Josèphe- $\mathrm{J}^{\prime}$ - Antoinette) (1802-1857) de -, fille $d^{\prime}$ Eugène : 30, $57-58,80,83$

- Libérat (Joseph-Eugène-) (1751-1832) de -, grand chât. de Sion, dép. à Zurich, trésorier d'Etat: 39-45, 47, 78, 86-87, $124,150-151,156,167,179,216,220$

- Louis (-Eugène-Ignace-Joseph) (18001874) de -, fils aîné d'Eugène, dit "Louis le Bon Garçon": 50, 59, 63, $67,72,74,79$

- Louis (Joseph-L'-Philippe-Guillaume Erasme) (1776-1842) de -, frère du comte Eugène, dit "Louis le Grand " : $35-36,48-50,53,58-59,63-64$

- Marie-Jeanne de -, née Duchemin, veuve de Joseph-Eugène-Adrien de Courten, épouse (1803) de Joseph-Antoine de Courten, belle-mère $d^{\prime}$ Eugène et de Pancrace : $30,53,64$

- Marie-Thérèse (1803-1865) de -, fille $d^{\prime}$ Eugène et $d^{\prime}$ Eugénie de Courten : 32

- Maurice (-Joseph-Jacques-Christophe) (1781-1847) de -, vice-prés. du gouvernement provisoire : $27,29,47-50$, $62,67,82,84-85,109,197-198$

- Pancrace (Jean-Joseph-Louis-Antoine-) (1774-1845) de -, frère $d^{\prime}$ Eugène : 26$27,29-38,46-85$

- Victor (Marie-Joseph-Antoine-V'-Fabien) $(1793-1855)$ de - : 34 


\section{D}

Dallèves, Alexis, lieutenant : 111, 113-114

Danesio, agent d'affaires des Courten: 35,58

Darbellay, Hyacinthe (1774-1852), bourgmestre de Monthey : 148, 195, 233

- Jean-Joseph, syndic de Collonges : 139

- Valentin, notaire, de Liddes, dép. à la Diète val. : 147-148

Daves, Jean-Joseph : 139

Day, banquier, à Paris : 36

Delasoie, Gaspard (-Etienne) (1768-1844), juge de paix d'Entremont, dép. à Fribourg-en-Brisgau : 25, 32, 109, 120, 122, 203

Délez, Claude, syndic de Salvan: 139, 161

Dessaix, Joseph-Marie (1764-1834), gén. français : 249

Diesbach, madame de - , sœur de Mme de Graffenried : 87

Dubois, Pierre-Louis, syndic de Dorénaz: 139

Dubosson, Hyacinthe, vice-prés. de Troistorrents : 195

Duc, Jean-Joseph (1748-1821) père, anc. grand chât. de Sion, dép. à Zurich : $71,81,89-90,159-160,163,185,187$, $189,213,216,218,223,227-228,231$

- Séverin (Jean-) († 1827), fils aîné de Jean-Joseph, syndic de Conthey, prés. du diz. de Conthey: 152-153

Du Fay, Pierre-Louis (1768-1843), anc. prés. du diz. de Monthey : 89, 138, $142,145,147-148,197,199-200,202$, $204,207-208,214-215,218$

Dufour, Marguerite (1767-1852), née $\mathrm{Du}$ Fay, épouse de Michel Dufour : 204

- Michel (1768-1843), anc. conseiller d'Etat, dép. à Zurich : 52, 56, 61-62, $65-66,69,73-75,77-78,80-81,86,89-$ $91,93-107,127,138,142,145,148,153$, $158-161,170,182,187,197,199,202$, $204,207-208,214,218,233,235,238-$ 267

\section{E}

Epp, Dominik, landamman d'Uri : 237

Escher, Hans-Konrad (1743-1814), bourgmestre de Zurich, prés. de la Diète fédérale : $65,67,81,84$

\section{$\mathbf{F}$}

Favre, Vincent, dép. d'Hérémence : 232 Fazakerley, F. N., ami de Canning, fondateur du Grillion's Club en $1813: 80-81$
Ferdinand (1781-1850), archiduc d'Autriche : 248

Fetzer, Karl, dép. d'Argovie à la Diète fédérale : 44, 240, 251

Finsler, Hans-Conrad (1765-1839), quartier-maître général : 188, 230, 244-245, 248-249, 255, 259-260, 267

Fischer, Friedrich, conseiller de légation de Berne à Zurich : 84

Flüe, Michael von -, landamman et dép. d'Obwald à la Diète fédérale : 84

Fournier, fournisseur de sel, à Lyon : 217

François Ier, empereur d'Autriche 18041835 : 28, 31-33, 40, 42-43, 105, 110, $114-115,118-119,174,257,262$

Frédéric-Guillaume III, roi de Prusse 1797$1840: 28,31,33-34$

Frédéric-Guillaume IV, fils aîné de Frédéric-Guillaume III : 34

Frédéric-Louis, $2^{\mathrm{e}}$ fils de Frédéric-Guillaume III : 34

Fuchs : 186

Füssli, Hans-Jacob (1766-1844), colonel, commandant de la $2^{\mathrm{e}}$ division : 248 , 250

Fux, Peter-Nikolaus (1743-1824), anc. grand chât. de Viège : 172

G

Gady, Nicolas (1766-1840) de -, colonel, commandant de la $1^{\text {re }}$ division : 255

Galland, v. Repingon

Gard, Frédéric (François-Joseph-) (17671849), anc. cap. de la bannière $d^{\prime} E n-$ tremont : 142, 145, 147-148

Gasser, Augustin (1766-1834), dép. de Fribourg à la Diète fédérale : 51

Gatschet, de Berne : 48

- madame -, de Berne : 58

Gattlen, Chrétien (1777-1866), de Rarogne, secrétaire : 173

Gay, Anne-Marie (1740-1815), née Du Fay, veuve de Jean-Joseph, lieutenant au service de France : 234

- Bruno (Joseph-) († 1830), anc. cap. au service du Piémont: 147-148, 192

- Emmanuel (1773-1842), médecin, anc. conseiller d'Etat: 138, 200, 204, 206$207,218-219,228,230,232$

- François-Joseph, syndic de Saxon : 147148

Gengel, Georg (1734-1821), dép. des Grisons à la Diète fédérale : 254

George III, roi d'Angleterre 1760-1820: 105

Gerstäcker, cap. autrichien : 119

Giroud, Joseph ( $\dagger$ av. 1829), collaborateur d'Isaac de Rivaz, à Saint-Maurice : 203 
Gottrau, Tobie de -, dép. de Fribourg à la Diète fédérale : 87

Graffenried, madame de -, sœur de Mme de Diesbach : 87

Griotti, Andreas, des Grisons, consul suisse à Trieste : 256

Guérin : 233

Guggelbiel, traiteur à Zurich : 237

Guillaume Ier, prince souverain des PaysBas 1813: 32

Guillot, Barthélemy (1754-1835), anc. cap. au service de France, secrétaire du conseil de diz. de Monthey : 148, 195, 208

H

Heer, Niklaus (1775-1822), landamman et dép. de Glaris à la Diète fédérale, commissaire général de la guerre : 44, 51, 255, 257

Hess, de Zurich : 237

Hirzel, Hans-Caspar (1751-1817), médecin, fondateur et prés. de la Société suisse d'utilité publique : 266

Hirzel de Saint-Gratien, chevalier, cap. au rég. de Roll : 50, 63, 70

\section{I}

Imhoff, Anton, de Conches : 171

Indermatten, Franz (-Joseph), dép. à $\mathrm{Zu}$ rich, grand chât. de Viège : 163, 171172,213

Ittig, Jean-Martin, lieutenant : 144

Ittner, Joseph-Albert (1754-1825) von -, ministre de Bade en Suisse 1807-1818 : 256

\section{J}

Jacquier, Jean-Baptiste (1769-1843), de Savièse, notaire : 62, 66, 204

Jean (1782-1859), archiduc d'Autriche : 251-252, 257, 262, 267

Joseph, domestique chez Eug. de Courten, à Sierre : 64

Jourdan, Jean-Baptiste, maréchal de France : 255

Julier, Augustin, grand chât. de Loèche : 173

- Etienne (Jean-) (1779-1829), chanoine de Sion : 197

- Franz, notaire, dép. à la Diète val. : 173

- Joseph (Jean-), trésorier d'Etat : 143

- Xavier, notaire, de Sierre: 81, 211
$\mathbf{K}$

Kaiser, Ludwig-Maria (1765-1840), landamman de Nidwald : 260

Kalbermatten, Louis (-Grégoire) (17681845) de -, dép. à Zurich : 74, 78, 8689

Kaufmann, Karl von -, ministre de Wurtemberg en Suisse : 56

Krüdener, Paul, baron de -, chargé d'affaires de Russie en Suisse 1815-1827 : $55-56,61,63,68-69,90,107,202$

Kuntschen, Alphonse (1787-1843), gendre de Léopold de Sépibus : 48-49, 54, 83 , 90

L

Labédoyère, Charles-Angélique-François Huchet (1786-1815), comte de -, gén. français : $264-265$

La Harpe, Frédéric-César (1754-1838) de -, homme d'Etat vaudois : 62

Lang, Casimir (1767-1815), anc. conseiller d'Etat, conseiller de préfecture : 24-25, 109, 172

Langeron, Gaspard-Louis (1772-1858), gén. français : 249

Laplane, Jean-Grégoire-Barthélemy Rouger (1766-1837), baron de -, gén. français : 249

Lavallaz, Antoine (1786-1870) de -, fils de Joseph, secrétaire du gouvernement provisoire : 73, 109, 111, 118, 121, 123

- Joseph (-Maurice) Du Fay (1758-1834) de -, maire de Sion : 24, 46-47

- Marie-Madeleine (1768-1832) de -, née de Courten, sœur d'Eugène et de Pancrace, épouse de Joseph de Lavallaz: 31,57

- Martin (1755-1832) de -, bénédictin à Einsiedeln 1784: 54-55, 89

- Pierre-Marie (1761-1834) de -, grand chât. de Monthey, dép. à Zurich : 3945, 47, 124, 162, 194-195, 200

Lecourbe, Claude-Jacques (1758-1815), gén. français : $245,247-248$

Liechtenstein, Aloys (1780-1833), gén. autrichien : 36

Liotard, Jean-Daniel, associé genevois d'Isaac de Rivaz: 203

Lise, mademoiselle : 53,58

Locher, Georges, et sa femme, logeurs, à Zurich : 201, 235, 237

Lorétan : 32

- Johann-Joseph (1760-1840), chât. de Loèche-les-Bains : 213

Louis XVIII, roi de France 1814-1824 : 48, $60,75,85,219,222,229-230,255,259-$ $260,263-265$ 
Luder, Pierre-François (1763-1830), grand chât. d'Entremont, bourgmestre de Sembrancher: 142, 145, 147-148, 153, 161

Lusser, Florian (1784-1824), chancelier et dép. d'Uri à la Diète fédérale : 237 . 240-241

Luxem, cap. autrichien : 111

\section{$\mathbf{M}$}

Macognin de la Pierre, Charles (-Melchior) (1783-1850), médecin, vice-bourgmestre de Saint-Maurice : $147-148$

Maillardoz, Antoine-Constantin (17651832), ministre de Suisse à Paris : 260

Mallet, Francis (1765-1839), baron, citoyen de Genève, gén. français : $67,75,87$, 218-219

Marcacci, Giovanni-Antonio (1769-1854), consul de Suisse à Milan : 247

Marchand, Jean-Gabriel (1765-1851), gén. français : 222-223

Marie-Louise, impératrice : 48, 59

Marulaz, Jacob-François Marola (17691842), dit -, gén. français : 255

Maurice, cap. non identifié : 58

Maximilien (*1782), archiduc d'Autriche : 245

Metternich, Clément, prince de -, ministre des Affaires étrangères et chancelier d'Autriche : 28-31, 33, 110, 119

Meyer von Schauensee, Franz-Bernhard (1763-1848), trésorier d'Etat et dép. de Lucerne à la Diète fédérale : 254

Michel, armurier, à Zurich : 250-251

Michel, "métral " (régisseur) chez les Courten, à Sierre : 35, 58, 67

Miollis, Sextius-Alexandre-François, gén. français : 223

Monod, Henri (1753-1833), dép. de Vaud à la Diète fédérale : 62

Montezuma, chevalier de - : 58, 81

Morand, Philippe (1773-1856), bourgmestre de Martigny, dép. à Zurich : 52, $56,61-62,65-66,73,78,89,93-107,138$, $140,142,145,148-149,153,159-160$, $166,170,182,190-191,197,213-214$, 229, 232-234

Morell, Johannes (1759-1835), landamman de Thurgovie, dép. à la Diète fédérale : 235

Mortier, Adolphe (1768-1835), maréchal de France : 31

Mottet, Claude : 139

Mousson, Jean-Marc (1776-1861), chancelier de la Confédération : 189, 229, 235, 239-240
Mülinen, Niklaus - Friedrich (1760-1833) von -, avoyer de Berne, dép. à la Diète fédérale : 62, 84, 235, 240-241, 262

Muralt, Hans-Conrad (1779-1869) de -, colonel : 250

Muret, Jules (1759-1847), dép. de Vaud à la Diète fédérale : 62

\section{$\mathbf{N}$}

Näf, commandant de bataillon d'Appenzell : 255

Napoléon Ier, empereur : 28, 30, 35-36, 82, 186, 188, 222-225, 229-231, 246-248, 255,264

Ney, Michel, maréchal de France : 265

Nucé, Benjamin de -, cap., secrétaire $\mathrm{d}^{\prime}$ Etat adjoint : 125, 129, 136

$\mathbf{O}$

Olbec, d'-, v. Tousard d'Olbec

Olry, Johann-Franz-Anton von -, ministre de Bavière en Suisse 1807-1827:85

Ott, Hans-Kaspar (1780-1856), lieutenantcolonel : 245, 252-253, 257, 261

\section{$\mathbf{P}$}

Paschoud-Rosset, Jean-François (17791838), entrepreneur de transports à Vevey et au Bouveret : 217

Perrig, Barthélemy, juge du diz. de Brigue : 144

- François-Xavier (1769-1825), anc. cap. au service de France : 232

Perruchoud, Chrétien, de Chalais : 81

Petitpierre, adjudant du gén. Marulaz: 255

Pfister, Balthasar (1757-1825), landamman et dép. de Schaffhouse à la Diète fédérale : 51,256

Pittier, Louis (Joseph-) (1753-1815), prés. $\mathrm{du}$ trib. de première instance et conseiller de préfecture, dép. à Zurich : 23-25, 62, 69, 71-75, 77-78, 81-83, 93$107,140,142,145,147-148,159-161$, 164-165, 175, 197, 204-207, 209

Pourtalès, lieutenant-colonel, commissaire en Valais : 239

Praroman, dép. de Fribourg à la Diète fédérale : 200

Preux, famille de - : 211

- Antoine (1768-1820) de -, banneret de Sierre : 163

- Joseph-Augustin de -, banneret de Sierre : 144 
- Joseph-Xavier de -, évêque de Sion 1807-1817: 46-47, 124, 127, 140, 169, $180,197,201,223,229$

- Louis (Pierre-) (1767-1852) de -, vicegrand chât. de Saint-Maurice : 138-139, $142,145,147-148,218$

Produit, Jean-Joseph, syndic de Leytron : $142,145,147-148$

Puy, René († 1820), négociant à SaintMaurice : 232

$\mathbf{Q}$

Quartéry, Jacques (1750-1826) de -, maire de Saint-Maurice, dép. à Fribourg-enBrisgau : 23, 25, 32, 89, 109, 139, 142, 145,218

$\mathbf{R}$

Rambuteau, Claude-Philibert Barthelot (1781-1869) de - préfet du département du Simplon : 23-24

Reinhard, Hans (1755-1855) von -, landamman de Zurich, prés. de la Diète fédérale : $39-45$

Rengger, Albrecht (1764-1835), dép. argovien au congrès de Vienne : 62

Repingon, Galland \& Cie, commis du sel à Genève : 25

Ribordy, Gabriel-Gaspard, notaire, chât. de Riddes : 228

- Jean-Pierre (1768-1837), de Liddes, anc. dép. à la Diète val. : 145, 147-148

Rickenmann, commandant de bataillon de Saint-Gall : 255

Riedmatten, Augustin (Joseph-) (17511837) de - , bourgmestre de Sion, dép. à Zurich : 75, 78, 86-88, 99, 163-164, 175

- Pierre-Joseph (1744-1812) de -, bourgmestre de Sion : 143

Rion, Antoine (1772-1814), notaire, substitut du procureur impérial : 25

Rivaz, Anne-Joseph (1751-1836) de frère d'Isaac, chanoine de Sion et historien : 219

- Benjamin (Gaspard-) (1783-1830) de -, fils aîné de Charles-Emmanuel : 87

- Charles (-Louis-Marie) (1796-1878) de ,$- 2^{\mathrm{e}}$ fils de Charles-Emmanuel : 86, 88-92

- Charles-Emmanuel (1753-1830) de -, anc. conseiller d'Etat, prés. de la commission du Bas-Valais, dép. à Zurich : 67, 72-78, 80-81, 86-92, 93-108, 138-140, 142, 145, 147-154, 156, 158-167, 170, $179-180,182,184-185,187,189-194$, 196-234
- Isaac (1752-1828) de -, conseiller de préfecture, membre du gouvernement provisoire : 24, 120, 124, 132-133, 137138, 153, 185, 197, 200-202, 204-206, 209-210, 213-214, 216, 218, 220-221, $223-225,228,230-231$

- Marie-Catherine-Julie (1759-1834) de - née de Nucé, épouse (1776) de Charles-Emmanuel : 86-92, 201

Roffler, Valentin (1777-1841), dép. des Grisons à la Diète fédérale : 254

Roll, rég. de $-: 63,67,70$

Roten, Aloys, anc. major de Rarogne, dép. à la Diète val. : 172

- Nicolas (François-) (1754-1839) père, major de Rarogne: 143

- Nicolas (1779-1830) fils, juge de paix de Rarogne, grand chât. de Rarogne : 24, 172

Rouyer, Nicolas-François, secrétaire de la légation française à Zurich : 87, 184

Ruchat, madame - à Bex : 87

Rüttimann, Vinzenz (1769-1844), avoyer et dép. de Lucerne à la Diète fédérale : $44,237,240-241,252,254,262$

\section{S}

Salis, madame de -, à Zurich : 64

Saudan, Pierre-Joseph, à Martigny : 211

Sauge, agent d'affaires, à Paris : 36

Schalen, Lucas (1766-1821) von -, anc. chât. de Viège : 144, 172

Schaller, K. L., auteur d'un Handbuch der deutschen Dicht- und Redekunst aus Beispielen entwickelt (Vienne, 1806, 2 vol.) : 50

Scheither, Georg (1772-1816), gén. autrichien : 250

Schmidtmeyer, Jean-Pierre (1768-1830), dép. de Genève à la Diète fédérale : 235,256

Schmiel, Johann-Nepomuk (1774-1850) von -, colonel, commandant de brigade : 255

Schöll, traiteur, à Zurich : 236-237

Schraut, Franz-Alban, baron de -, ministre d'Autriche en Suisse 1807-1825: $40,42-43,47,68,72,76-78,81-82,86-$ $87,90,107,114-115,118,123,146$, $150-151,153-154,159-160,166,174-$ 175, 182, 184, 200, 202, 218-219, 227, 229-230, 235-236, 238, 253

Schulthess-Rechberg, Barbara (1743-1818), à Zurich : 64

Schwarzenberg, Charles-Philippe, duc de Kruman, prince de -, généralissime de la Coalition : 25, 27, 29-30, 119, 245$246,248,267$ 
Secrétan, Louis (1758-1839), dép. de Vaud à la Diète fédérale : 62

Sépibus, Casimir (1757-1842) de -, notaire, secrétaire allemand de la commission de constitution : 24, 127

- Léopold (1759-1832) de -, prés. de la Diète val., prés. de la commission de constitution, dép. à Zurich : 39-47, 49$52,54-57,60-63,65-66,68-70,73-78$, $80,82-83,86-90,94,103,124,127,136$, $144,149,151,154,156,159-160,163$, $165-166,168-173,177-179,187-189,192$, 205, 209-211, 216-217, 219-220, 223, 236, 243

Siegfried, commandant de bataillon d'Argovie : 255

Sierro, Jean, d'Hérémence, dép. à Zurich : 81,90

Sigristen, Jacob-Valentin ( $†$ 1808), dernier grand bailli de l'ancien régime: 99, 143

Simbschen, Joseph-Franz (1781-1824) von -, colonel commandant du détachement autrichien en Valais : 24-25, 27, 29, 31, $36,42,108-109,111-113,115-116,118-$ 123,210

Sonnenberg, Ludwig (1782-1850) von -, colonel fédéral : 245

Steffen, Augustin, juge du diz. de Conches : 143, 171

Steigentesch, August (1774-1826), baron von -, major-gén. autrichien : 235$236,239,243-246,249,252-253$

Steininger, gén. autrichien : 260

Stockalper, Gaspard-Eugène (1750-1826) de $\rightarrow$, baron, conseiller à la cour de Lyon, dép. à Fribourg-en-Brisgau, dép. à la Diète fédérale : 24-25, 27, 30, 32-33, 109, 111, 119-122, 235-267

Stockmann, Josef-Ignaz (1762-1837), landamman et dép. d'Obwald à la Diète fédérale : 237,260

Stürler, Johann-Rudolf (1771-1861), dép. de Berne à la Diète fédérale : 84

Suchet, Louis-Gabriel (1770-1826), maréchal de France : 245

T

Tabin, Mathias (* 1753), d'Anniviers : 81

Taffiner, Jean-François (1756-1844), viceprés. exerçant les fonctions de prés. du gouvernement provisoire : 49, 51, 62, $69-70,73,80-81,83,129,133,136,144$, 197-198, 203, 205-207, 210-211

Talleyrand, Auguste de -, ambassadeur de France en Suisse 1808-1823: 23, 56, $60-61,64-65,67,87,151,184,235,257$, 260
- Charles-Maurice, ministre des Affaires étrangères de France : 263

- Louis-Marie-Anne, baron, diplomate, père $d^{\prime}$ Auguste : 60

Tanner, Konrad, abbé d'Einsiedeln 1808I825: 54-55

Theiler, du Simplon : 230

Tissières, voiturier, à Martigny : 86

Toggenburg, Paul-Anton (1770-1824), chef de bataillon des Grisons : 255

Tousard d'Olbec, Anne-Louise, fille de Louis : 23

- Catherine, fille de Louis : 87

- Louis (-Anne-François) (1757-1840), directeur des Finances du département du Simplon : 23, 91

- Marguerite (1761-1841), née de Nucé, épouse de Louis : 91-92

Trombert, Barthélemy, de Val-d'Illiez, anc. chât. : 142, 145, 148, 195

Tschann, Georges (1777-1847) de - , chargé d'affaires suisse à Paris : 260, 263265

Turreau de Garambouville, Louis-Marie (1756-1816), commandant des troupes françaises en Valais 1799-1802 : 39, 42, 74,200

\section{$\mathbf{U}$}

Ugarte, Aloys (1784-1845), comte d'-, gouverneur civil autrichien à Genève : 122

Usteri, Paul (1768-1831), dép. de Zurich à la Diète fédérale : 235,256

\section{V}

Van der Hoeven, Elie, ministre des PaysBas en Suisse 1814-1817: 252

Varax, François, comte de -, ministre de Sardaigne en Suisse 1815: 217, 235236

Victor-Emmanuel Ier, roi de Sardaigne 1802-1821: 29, 58, 80-81, 85

Vom Brüel, Louis, conseiller, à Monthey : 195

Vukassovich, Philipp, major sous les ordres de Simbschen : 122

\section{W}

Walker, Adrien (1785-1837), de Mörel, dép. à la Diète val. : 163, 171, 213

Wallmoden, Ludwig-Georg-Thedel (17691862) von - , gén. autrichien : 248

Walpen, Joseph-Ignaz, vice-grand chât. de Conches : 171 
Watteville, rég. de - : $\mathbf{7 2}$

- Abraham-Ludwig-Karl (1776-1836) de -, gén. au service d'Angleterre : 72

- Nicolas-Rudolf (1760-1832) de -, gén., avoyer de Berne : 26, 35, 38

Wegener, Maurice-Joachim (1742-1818), grand cap. de Brigue : 213

Weger, Dominique (1758-1828), cap. en Piémont, prés. du diz. de Conches : 170, 213

Wellesley, Richard ( +1831 ), fils de lord R. Colley $W^{\prime}$ (1760-1842) : 80-81

Wellington, Arthur Wellesley (1769-1852), duc de -, gén. anglais : 244

Werra, Ferdinand (1770-1824) de -, baron, dép. à Fribourg-en-Brisgau : 24$25,32,109,120,122,228$

- Ignaz (Franz-) (1768-1842) de -, prés. du diz. de Loèche, secrétaire allemand de la Diète val. : 173

- Meinrad (1782-1841) de -, neveu de Charles-Emmanuel de Rivaz : 87

Wieland, Johannes, officier d'artillerie, fils du bourgmestre : 266
- Johann-Heinrich (1758-1838), bourgmestre et dép. de Bâle à la Diète fédérale : 240, 266

Würsch, Franz-Xaver, landamman de Nidwald : 260

Wurstemberger, Johann-Ludwig (1783-1862) von -, préfet de Frutigen : 115, 189

Wyss, David (1763-1839) von -, bourgmestre de Zurich, prés. de la Diète fédérale : 165-166, 168, 172, 174-176, 179, 188-190, 229-230, 235-236, 238-241, 243244, 247-248, 250, 253-254, 256-257, $259,262-264$

Z

Zelger, Franz-Nikolaus (1765-1821), landamman de Nidwald : 260

Zellweger, Jakob (1770-1821), landamman et dép. d'Appenzell à la Diète fédérale : 44, 240, 245, 251-252

Zen Ruffinen, Alexis (1767-1829), vicegrand chât. de Loèche : 173

- François-Joseph, percepteur des contributions, grand chât. de Loèche : 24 , 210-211 